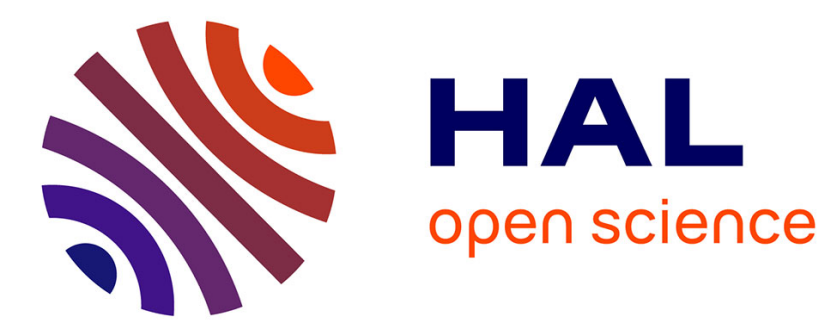

\title{
Power Spectrum density of Single Side band CPM using Lorenztian frequency pulses
}

\author{
Haïfa Farès, D. C Glattli, Yves Louët, Jacques Palicot, Preden S Roulleau,
} Christophe Moy

\section{> To cite this version:}

Haïfa Farès, D. C Glattli, Yves Louët, Jacques Palicot, Preden S Roulleau, et al.. Power Spectrum density of Single Side band CPM using Lorenztian frequency pulses. IEEE Wireless Communications Letters, 2017, 6 (6), 10.1109/LWC.2017.2742505 . hal-02087905

\section{HAL Id: hal-02087905 https://hal.science/hal-02087905}

Submitted on 2 Apr 2019

HAL is a multi-disciplinary open access archive for the deposit and dissemination of scientific research documents, whether they are published or not. The documents may come from teaching and research institutions in France or abroad, or from public or private research centers.
L'archive ouverte pluridisciplinaire HAL, est destinée au dépôt et à la diffusion de documents scientifiques de niveau recherche, publiés ou non, émanant des établissements d'enseignement et de recherche français ou étrangers, des laboratoires publics ou privés. 


\title{
Power Spectrum density of Single Side band CPM using Lorenztian frequency pulses
}

\author{
Haïfa Fares ${ }^{2}$, D. Christian Glattli ${ }^{1}$, Yves Louet ${ }^{2}$, Jacques Palicot ${ }^{2}$, Preden Roulleau ${ }^{1}$, and Christophe Moy ${ }^{2}$ \\ ${ }^{1}$ SPEC UMR 3680 CEA-CNRS,Université Paris-Saclay, CEA-Saclay, 91191 Gif-Sur-Yvette, France \\ ${ }^{2}$ IETR UMR CNRS 6164 - CentraleSupélec , Avenue de la Boulaie, 35557 Cesson-Sévigné, France
}

\begin{abstract}
We propose a new waveform for frequency-shift keying continuous phase modulation (FSK-CPM) that directly generates a single side band (SSB) spectrum. The original principle, inspired from quantum physics (levitons), is presented. From the simple analytical form of the modulation, orthonormal wave-functions and analytical expressions leading to the SSB power spectrum density are proposed. This CPM modulation displays a very compact spectrum, with $99 \%$ of the power in the primary bandwidth. Its SSB property makes this FSKCPM scheme, hereby called SSB-FSK, a promising solution not sensitive to image frequency degradation and would allow large bit rates transmission at low carrier frequency.
\end{abstract}

\section{INTRODUCTION}

Spectrum scarcity has been driving most of studies for decades in telecommunication area so as to design more and more well frequency localized waveforms. Given that data rates to be transmitted could be large, spectrum efficiency is the first parameter to be considered (and to be maximized in some contexts). In parallel, energy consumption of ICT (Information and Communication Technology) has became a major concern for all the community and ways to mitigate its carbon footprint have to be investigated. This leads to the second parameter to be considered for any transmissions namely its energy efficiency. Waveforms and transmission schemes behave differently regarding these efficiencies depending on the context: for instance, post Orthogonal Frequency Division Mutliplexing (OFDM) waveforms intended for further 5G mobile broadband networks [1] have very high spectrum efficiency at the expense of the energy efficiency due to the non constant envelope of the transmitted signal what leads to an over consumption of the high power amplifier [2]. At the opposite, Continuous Phase Modulations (CPM) family has a quite low spectral efficiency but on the other hand its energy efficiency is very high since the transmitted signal has a constant envelope and can consequently be amplified very close to the saturation point of the power amplifier where its efficiency is maximum.

In this context the proposed study is focused on a constant envelope FSK-CPM waveform whose frequency pulse has the property to directly generate a narrow single side band (SSB) spectrum. This proposed scheme will be referred as SSBFSK in the remainder of the paper. Contrary to regular SSB

This work was supported by the ERC Proof of Concept 680875 CLevitonics schemes [3], it does not need any additional post-modulation filter to generate the single side band spectrum what constitutes the originality of the proposed transmission scheme. This frequency pulse (known as a Lorentzian) originates from quantum physics ideas (levitons, see [4]) and is applicable to classical signals but it has never been applied to digital communications. This paper intends to give some theoretical derivations related to the power spectrum density of the transmitted signal. The content of the paper is as follows. First, we introduce the principle of SSB modulation in Section II. Then, the power spectral density of the proposed modulation is derived in Section III. Simulation results corroborate the tightness of the analytical derivations.

\section{Principle of the Single Side Band Continuous PHASE MODULATION}

We seek for a constant amplitude signal $s(t)=\cos \left(2 \pi f_{c} t+\right.$ $\phi(t))$ where $f_{c}$ is the carrier frequency whose phase variation $\phi(t)$ aims at providing a single side band spectrum. We first consider a single symbol per frequency pulse and give the mathematical derivation leading to the generic frequency pulse $g(t)=d \phi(t) / d t$ giving SSB property.

\section{A. Single symbol case}

To proceed we consider the baseband representation $s_{l}(t)$ of the modulated waveform $s(t)$ given by $\exp (j \phi(t))$. Its Fourier transform is

$$
\mathcal{F}\left[s_{l}(t)\right]=S_{l}(f)=\int_{-\infty}^{\infty} s_{l}(t) \exp (-j 2 \pi f t) d t .
$$

Using integration in the complex plane $z=t+j t^{\prime}$, (1) can be written as

$$
S_{l}(f)=\oint_{\gamma^{ \pm}} s_{l}(z) \exp (-j 2 \pi f z) d z
$$

where $\gamma^{+}\left(\gamma^{-}\right)$is a closed contour running from $-\infty$ to $+\infty$ followed by a semi-circle with infinite radius situated in the upper (lower) plane for positive (negative) frequency respectively. The necessary condition to have a finite value for $f>0$ and zero value for $f<0$ is that $s_{l}(z)$ has at least one pole in the upper and no pole in the lower complex plane. The simplest and unique mathematical form of $s_{l}(t)$ ensuring these conditions is

$$
s_{l, 0}(t)=\frac{t-t_{0}+j w_{0}}{t-t_{0}-j w_{0}}=\exp \left(j \phi_{0}(t)\right)
$$


where the pole has been arbitrarily chosen as $z=t_{0}+j w_{0}$. This leads to the generic levitonic phase term $\phi_{0}(t)=$ $\pi+2 \arctan \left(\left(t-t_{0}\right) / w_{0}\right)$ where we have set $\phi(-\infty)=0$. Its derivative gives the elementary frequency pulse $g_{0}(t)$ expressed by

$$
g_{0}(t)=\frac{2 w_{0}}{\left(t-t_{0}\right)^{2}+w_{0}^{2}} .
$$

This is a Lorentzian centered at $t_{0}$ with half width at mid height equal to $w_{0}$. The baseband power spectrum is $\left|S_{l}(f)\right|^{2}=4 \pi w_{0} H(f) \exp \left(-4 \pi w_{0} f\right)$ where $H($.$) is the Heav-$ iside step function expressing the SSB property. Importantly, the phase increment $\Delta \phi_{0}$ corresponds to a modulation index $h=1$ and is given by $\int_{-\infty}^{\infty} g_{0}(t) d t=2 \pi$.

\section{B. Binary single side band frequency shift keying : 2-SSB-FSK}

Multiple Single Side Band pulses can be similarly generated by introducing new poles in the upper complex plane, generalizing Eq.(3) to

$$
s_{l}(t)=\prod_{k=1}^{N} \frac{t-t_{k}+j w_{k}}{t-t_{k}-j w_{k}}=\prod_{k=1}^{N} s_{l, k}(t) .
$$

This corresponds to a sum of Lorentzian pulses $g_{k}(t)$ with

$$
\frac{d \phi(t)}{d t}=\sum_{k=1}^{N} g_{k}(t)=\sum_{k=1}^{N} \frac{2 w_{k}}{\left(\left(t-t_{k}\right)^{2}+w_{k}^{2}\right)},
$$

where the width $w_{k}>0$ and pulse time $t_{k}$ of each elementary pulse $g_{k}(t)$ can be arbitrarily chosen. The Fourier Transform of the total signal $s_{l}(t)$ is given by the convolution product $S_{l}=S_{l, 0} * S_{l, 1} * S_{l, 2} * \ldots * S_{l, N}$ of the Fourier transforms $S_{l, k}$ of each elementary $s_{l, k}(t)$. Being the convolution of SSB spectra with positive frequencies, the outcome is also a SSB spectrum with positive frequencies. This is consistent with the absence of pole in the lower half complex plane. Note that changing the sign of $\phi(t)$ (modulation index $h=-1$ ) is like switching all poles from the upper to the lower plane and provide a mirror single side band spectrum with only negative frequencies.

A particular example is a periodic series of Lorentzian pulses of period $T$ and pulse width $w$

$$
g(t)=\sum_{k=-\infty}^{+\infty} \frac{2 w}{(t-k T)^{2}+w^{2}}=\frac{\pi}{T} \frac{\sinh \left(2 \pi \frac{w}{T}\right)}{\sin \left(\pi \frac{t}{T}\right)^{2}+\sinh \left(\pi \frac{w}{T}\right)^{2}}
$$

giving the complex base band signal

$$
s_{l}(t)=\frac{\sin (\pi(t+j w) / T)}{\sin (\pi(t-j w) / T)} .
$$

Its Fourier Transform is a series of Dirac peaks with only positive frequencies $f_{l}=l / T, l \geq 0$. The Fourier amplitudes $p_{l}$ have been calculated in [5] in the context of quantum physics and are given by : $p_{0}=-\beta, p_{l \geq 1}=\left(1-\beta^{2}\right) \beta^{(l-1)}$ where $\beta=\exp (-2 \pi w / T)$.

After having shown that an arbitrary sum of Lorentzian pulses keeps the SSB spectrum property, we are ready to apply these levitonic pulses to a new type of FSK-CPM with the unprecedented Single Side Band property leading to SSB-FSK.
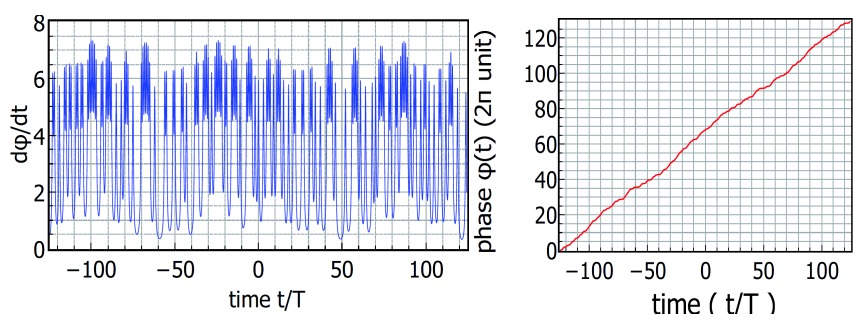

Fig. 1. Left panel: simulation of a 248 bit sequence of levitonic phase pulses; right panel: integrated phase. Each phase pulse gives $2 \pi$ increment (as the modulation index $h=1$ )

Let $\left\{\mathbf{b}_{k}\right\}$ a RZ binary sequence of bits $\left(b_{k}=1,0\right)$ and write the phase derivative

$$
\frac{d \phi(t)}{d t}=g(t)=\sum_{k=-\infty}^{+\infty} \frac{b_{k} \cdot 2 w}{(t-k T)^{2}+w^{2}} .
$$

A numerical example, using a pseudo-random binary sequence of $b_{k}$ is given in Fig.1, left panel, and the corresponding integrated phase in the right panel. The base band signal is

$$
s_{l}(t)=\exp (j \phi(t))=\prod_{k=1}^{N}\left(\frac{t-k T+j w}{t-k T-j w}\right)^{b_{k}} .
$$

In practice, synthesizing digitally the modulated phase can be done by first generating the sum of the frequency pulses from (9) followed by numerical integration or by directly synthesizing the phase $\phi(t)=\sum_{k} b_{k} 2 \arctan ((t-k T) / w)$. The periodic case (Eq. (7),(8)) corresponds to $b_{k}=1$ for all $k$.

An example of Power Spectral Density (PSD) obtained after averaging several power spectra of simulated high frequency signal $s(t)=\cos \left(2 \pi f_{c} t+\phi(t)\right)$ for independent pseudorandom series of equiprobable $b_{k}$ is given in Fig.3, left panel. It is compared with the theoretical spectrum, right panel, whose derivation is given below in section III. The time unit is the symbol emission period $T=1$. The carrier frequency is $f_{c}=10 / T$ and the frequency pulse width is $w=0.37 T$.

\section{Example of Quaternary modulation : 4-SSB-FSK}

In this section we generalize the binary frequency pulse modulation given by Eq.(9) to a $\mathrm{N}$-ary modulation where the bits $\left\{\mathbf{b}_{k}\right\}$ take $N=2^{p}$ values with $b_{k}=0,1,2, \ldots, 2^{p}-1$. A first question is: does multilevel modulation keeps SSB property ? To answer, let us first consider a single pulse. Looking at Eq.(3), we see that multiplying the elementary phase pulse centered at $t_{0}$ by an integer $h=K$ (i.e. $\phi(t)=K \phi_{0}(t)$ ) leads to :

$$
\exp (j \phi(t))=\left(\frac{t-t_{0}+j w_{0}}{t-t_{0}-j w_{0}}\right)^{K} .
$$

This expression exhibits a pole of order $K$ in the upper-half complex plane while it still lacks of pole in the lower half complex plane preserving the SSB property. The modulation index is now $h=K$. The generation of a multi-level 

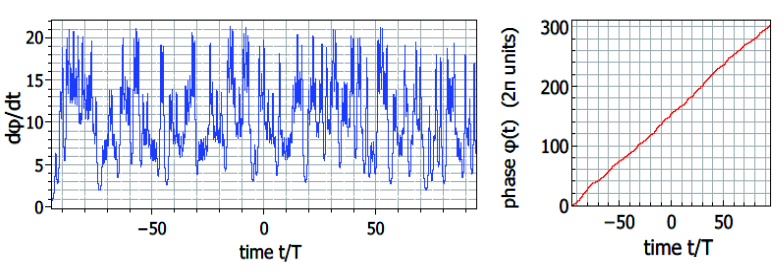

Fig. 2. Left panel: simulation of a 189 bit sequence of levitonic quaternary phase pulses; right panel: integrated phase.

modulation like a quaternary modulation can be performed by making the product of a binary modulation signal $s_{l, 1}(t)$, with $h=1$ and bits $b_{k, 1}=0,1$, by a second binary modulation signal $s_{l, 2}(t)$, with $h=2$ and bits $b_{k, 2}=0,1$, giving

$$
s(t)=s_{l, 1}(t) s_{l, 2}(t)=\prod_{k=1}^{N}\left(\frac{t-k T+j w}{t-k T-j w}\right)^{b_{k, 1}+2 b_{k, 2}} .
$$

The corresponding quaternary frequency pulses gives

$$
\frac{d \phi(t)}{d t}=\sum_{k=-\infty}^{+\infty} \frac{\left(b_{k, 1}+2 b_{k, 2}\right) \cdot 2 w}{(t-k T)^{2}+w^{2}} .
$$

Figure 2 shows a numerical simulation of quaternary phase pulses and the integrated phase. Being the convolution of two SSB spectra the spectrum of $s(t)$ keeps the SSB property. The same reasoning generalizes to higher multi-level modulations.

A second issue concerns the practical use of a multilevel modulation. It is important to know whether the waveforms for different integers $h$ are orthogonal. Indeed, the orthogonality would ease the demodulation at the receiver.

To show this, lets consider the single pulse signal $s_{l, n}(t)=$ $\left(\frac{t-t_{0}+j w}{t-t_{0}-j w}\right)^{n}=\exp \left(j n \phi_{0}(t)\right)$ corresponding to an integer value $n$ of the $\mathrm{N}$-ary bits. One can write a scalar product showing the orthogonality of two signals differing by two bit values $n$ and $m$ in the following way. Starting from the identity

$$
\frac{1}{2 \pi} \int_{-\pi}^{+\pi} e^{j(n-m) \phi_{0}} d \phi_{0}=\delta_{n, m}
$$

and using the change of variable: $\phi_{0}(t)=2 \arctan ((t-$ $\left.\left.t_{0}\right) / w\right)$, Eq. (14) becomes

$$
\frac{1}{2 \pi} \int_{-\infty}^{+\infty} s_{l, m}(t)^{*} s_{l, n}(t) g_{0}(t) d t=\delta_{n, m}
$$

where the generic Lorentzian pulse $g_{0}(t)$ given by Eq.(4) appears as a weight function ensuring orthogonality of the two waveforms. Thus, our proposed SSB modulation shows the simple property of generating orthogonal waveforms by simply multiplying by an integer the generic levitonic phase $\phi_{0}$. In order to characterize the $\mathrm{N}$-ary SSB-FSK modulation the next step is to derive its power spectral density. As the frequency shift is larger for $b_{k}$ larger than 1 , one expects the spectrum to be broader than the binary SSB-FSK. Fig.5 shows the numerical simulation of the PSD for quaternary modulation (4-SSB-FSK).

\section{Power Spectral Density Derivation}

Deriving the Power Spectral Density (PSD) of phase modulated signals is usually a difficult task and in some cases such expression does not exist. Fortunately, the simple form of the elementary waveform (see Eq. (3)) enables the analytical computation of the statistical average of the two-time correlation function of the low pass signal. Then, the numerical Fourier Transform of its time average $C(\tau)=\frac{1}{T} \int_{0}^{T} E\left[s_{l}(t)^{*} s_{l}(t+\right.$ $\tau)] d t$ gives the PSD $P(f)=\int_{-\infty}^{\infty} C(\tau) \exp (j 2 \pi \tau) d \tau$.

\section{A. Binary SSB-FSK}

To proceed, we will set $T=1$ and consider $\left\{\mathbf{b}_{k}\right\}$ as uncorrelated Bernouilli random variables with probability $P\left[b_{k}=1\right]=p$ and $P\left[b_{k}=0\right]=1-p$. For ease in computation the time delay $\tau$ in the two-time correlation function is symmetrized as

$$
C\left(t-\frac{\tau}{2}, t+\frac{\tau}{2}\right)=\prod_{k=-\infty}^{\infty}\left(\frac{t_{k}-\frac{\tau}{2}-j w}{t_{k}-\frac{\tau}{2}+j w} \cdot \frac{t_{k}+\frac{\tau}{2}+j w}{t_{k}+\frac{\tau}{2}-j w}\right)^{b_{k}}
$$

where $t_{k}=t-k$. After averaging over statistically equivalent bit sequences, we get

$$
\begin{aligned}
E\left[C\left(t-\frac{\tau}{2}, t+\frac{\tau}{2}\right)\right] & =\prod_{k}\left(1-p+p \frac{t_{k}^{2}-\left(j w+\frac{\tau}{2}\right)^{2}}{t_{k}^{2}-\left(j w-\frac{\tau}{2}\right)^{2}}\right) \\
& =\frac{\sin \left(\pi ( t - \theta _ { p } ( \tau ) ) \operatorname { s i n } \left(\pi\left(t+\theta_{p}(\tau)\right)\right.\right.}{\sin \left(\pi ( t - j w + \frac { \tau } { 2 } ) \operatorname { s i n } \left(\pi\left(t+j w-\frac{\tau}{2}\right)\right.\right.}
\end{aligned}
$$

where $\theta_{p}(\tau)=\sqrt{\frac{\tau^{2}}{4}-w^{2}-(1-2 p) j w \tau}$. Note that Eq.(17) interpolates between no modulation $\left(p=0\right.$, all $\left\{b_{k}=0\right\}$ and $C$ is constant) and the periodic case $(p=1)$.

Eq.(17) shows that the two-time correlation function is periodic in the mean time $t$. Indeed, cyclo-stationary property is expected for a regular bit period emission. Its time average gives the correlation function required for the calculation of the PSD. Its expression for the usual case $(p=1 / 2)$ is

$$
C(\tau)=1+\frac{\cos \left(\pi \sqrt{\tau^{2}-4 w^{2}}\right)-\cos (\pi(\tau-2 j w))}{\sin (\pi(\tau-j 2 w))} .
$$

The first term in the numerator describes the continuous spectrum expected from the random symbol emission. The second term, combined with the denominator, is periodic and is responsible for narrow peaks in the spectrum. This is expected for integer modulation index values. The denominator is a complex sine function which displays poles only in the upper half complex plane and ensures single side band property. The continuous spectrum in the denominator is numerically found to take non zero values on a frequency interval of width $1 / T$. From Fig. 3 the spectrum can be viewed as a main spectrum shape located in the range $0 \leq f-f_{c} \leq 1 / T$ with $1 / T$ frequency shifted replica, each exponentially attenuated by $\exp (-4 \pi w / T)$. For $w / T=0.37$ this corresponds to $-20 \mathrm{~dB}$ for every $1 / T$ frequency shift.

Figure 4 shows the integrated power starting from the carrier frequency. For $w / T=0.37,99 \%$ of the power falls in a bandwidth $B=1 / T$ suggesting that a good spectral efficiency can be expected. For a smaller pulse width $(w=0.19) \quad 90 \%$ of the power is located in a $1 / T$ bandwidth. 

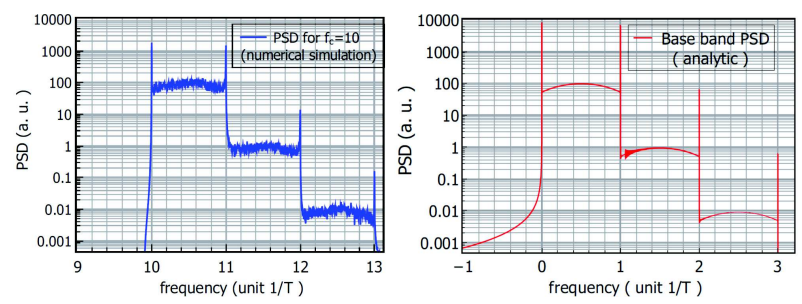

Fig. 3. Power Spectral Density (PSD) of binary Single Side Band phase shift Keying with pulse width $w=0.37 T$. Left: numerical simulation corresponding to the average of 32 spectra of 248 independent binary sequence of phase pulses. Right : PSD from Numerical Fourier transform of the analytical expression (18). The spectrum is made from a main part located in the $[O, 1 / T]$ frequency interval, followed by $1 / T$ frequency shifted replica, each attenuated by the ratio $\exp (-4 \pi w / T)(-20 \mathrm{~dB}$ here).

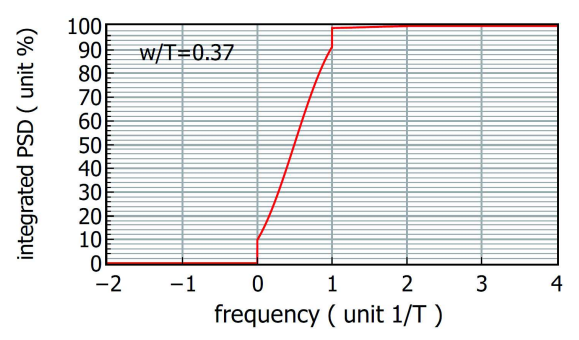

Fig. 4. Integrated Power Spectral Density (PSD) of binary Single Side Band phase shift Keying with pulse width $w=0.37 T$. One sees that $99 \%$ of the power in the bandwidth $B=1 / T$

\section{B. Quaternary SSB-FSK}

A calculation of the two-time correlation function is also analytically possible. For a quaternary modulation, the twotime correlation function for uncorrelated equiprobable bits $b_{k}=0,1,2,3$ is given by

$$
E\left[C\left(t-\frac{\tau}{2}, t+\frac{\tau}{2}\right)\right]=\frac{\prod_{k=0}^{k=2}\left(\cos 2 \pi \theta_{k}(\tau)-\cos 2 \pi t\right)}{(\cos 2 \pi(\tau / 2+j w)-\cos 2 \pi t)^{3}}
$$

where $\theta_{0}(\tau)=\sqrt{\frac{\tau^{2}}{4}-w^{2}}, \theta_{1}(\tau)=\sqrt{\frac{\tau^{2}}{4}-w^{2}-w \tau}$, and $\theta_{2}(\tau)=\sqrt{\frac{\tau^{2}}{4}-w^{2}+w \tau}$. Analytical calculation of the time
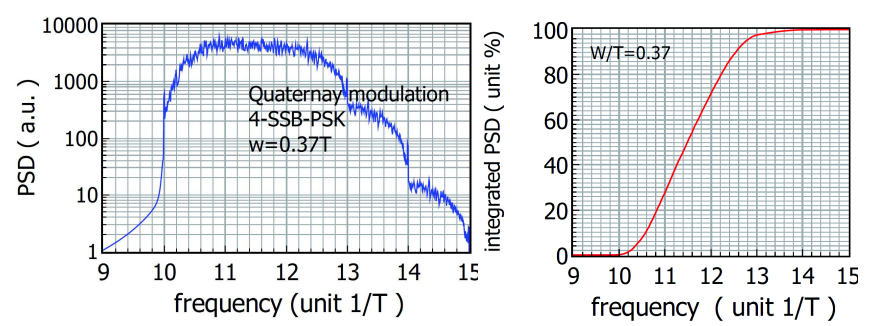

Fig. 5. Left panel: Power Spectral Density (PSD) of Quaternary Single Side Band frequency shift Keying (4-SSB-FSK) with phase pulse width $w=$ $0.37 T$. Right panel : integrated PSD. One sees that $98 \%$ of the power lies in the bandwidth $B=3 / T$ average of this expression is possible using standard complex integration. The expression is cumbersome and will not be given here for limited space. Its numerical Fourier Transform gives a PSD in perfect agreement with the simulations of Fig.5.

\section{CONCLUSION}

We have shown that the frequency pulses giving rise to SSB-FSK are Lorentzian (levitonic) frequency pulses. The modulation index must be integer (i.e. phase increments is multiple of $2 \pi$ ) and of same sign to provide SSB property. Non integer phase pulses restore a double Side Band spectrum as $\exp (j \phi(t))$ lacks of analyticity. Reversing the sign of the phase pulse gives a mirror spectrum (upper and lower side bands are interchanged). Like GMSK, SSB-FSK is a continuous phase modulation with narrow spectrum where almost all power falls in a bandwidth $1 / T$, i.e. the symbol rate. Beyond the capabilities of GMSK, SSB-FSK is able to provide multilevel modulation.

We have not discussed the demodulation issues. As for GMSK, SSB-FSK suffers from interference between symbols as the tails of the Lorentzian have significant overlap. A first approach for demodulation have been proposed in [6]. Efficient demodulation schemes have been developed in [7] using truncated frequency pulses and the Bit Error Rate (BER) in perfect channels (additive gaussien) has been evaluated.

We claim that the most original aspect brought by SSBFSK lies in its single side band property. In particular, the total absence of lower side band allows to operate with symbol rate $B=1 / T$ much larger than the carrier frequency $f_{c}$, typically $B=4 f_{c}$ without deterioration of the BER as there is no interference with the mirror spectrum.

\section{REFERENCES}

[1] Behrouz Farhang-Boroujeny, Hussein Moradi "OFDM Inspired Waveforms for 5G" IEEE Communications Surveys and Tutorials Volume: 18 Issue: 42016

[2] X. Yu, T. Wild, F. Schaich "Impact of RF transmitter hardware on 5G waveforms: Signal conditionings for UF-OFDM" Wireless Communication Systems (ISWCS)Symposium, 2016

[3] Arthur A. Oswald Early History of Single-Sideband Transmission Proceedings of the IRE Volume: 44 Issue: 12 Dec. 1956

[4] "Minimal-excitation states for electron quantum optics using levitons", J. Dubois, T. Jullien, F. Portier, P. Roche, A. Cavanna, Y. Jin, W. Wegscheider, P. Roulleau and D. C. Glattli, Nature 502, 659663 (2013)

[5] J. Dubois, T. Jullien, C. Grenier, P. Degiovanni, P. Roulleau, and D. C. Glattli, Phys. Rev. B 88, 085301 (2013).

[6] D.C. Glattli and P. Roulleau, patent WO2016124841 A1, 2016.

[7] Hafa Fares, Christian Glattli, Yves Louet, Christophe Moy, Jacques Palicot, Preden Roulleau "New Binary Single Side Band CPM", ICT 2017, Cyprus, May 2017 